\title{
Differences in Callosal and Forniceal Diffusion between Patients with and without Postconcussive Migraine
}

\author{
(D) L.M. Alhilali, (D). Delic, and (D) S. Fakhran
}

\begin{abstract}
BACKGROUND AND PURPOSE: Posttraumatic migraines are common after mild traumatic brain injury. The purpose of this study was to determine if a specific axonal injury pattern underlies posttraumatic migraines after mild traumatic brain injury utilizing Tract-Based Spatial Statistics analysis of diffusion tensor imaging.
\end{abstract}

MATERIALS AND METHODS: DTI was performed in 58 patients with mild traumatic brain injury with posttraumatic migraines. Controls consisted of 17 patients with mild traumatic brain injury without posttraumatic migraines. Fractional anisotropy and diffusivity maps were generated to measure white matter integrity and were evaluated by using Tract-Based Spatial Statistics regression analysis with a general linear model. DTI findings were correlated with symptom severity, neurocognitive test scores, and time to recovery with the Pearson correlation coefficient.

RESULTS: Patients with mild traumatic brain injury with posttraumatic migraines were not significantly different from controls in terms of age, sex, type of injury, or neurocognitive test performance. Patients with posttraumatic migraines had higher initial symptom severity $(P=$ .01) than controls. Compared with controls, patients with mild traumatic brain injury with posttraumatic migraines had decreased fractional anisotropy in the corpus callosum $(P=.03)$ and fornix/septohippocampal circuit $(P=.045)$. Injury to the fornix/septohippocampal circuit correlated with decreased visual memory $(r=0.325, P=.01)$. Injury to corpus callosum trended toward inverse correlation with recovery $(r=-0.260, P=.05)$.

CONCLUSIONS: Injuries to the corpus callosum and fornix/septohippocampal circuit were seen in patients with mild traumatic brain injury with posttraumatic migraines, with injuries in the fornix/septohippocampal circuit correlating with decreased performance on neurocognitive testing.

ABBREVIATIONS: $C C=$ corpus callosum; FA = fractional anisotropy; FSHC = fornix/septohippocampal circuit; 5-HT = serotonin; $m$ TBI = mild traumatic brain injury; PTH = posttraumatic headache; PTM = posttraumatic migraine

$\mathbf{M}^{\mathrm{i}}$ ild traumatic brain injury (mTBI), often referred to as "concussion," affects nearly 42 million individuals worldwide, annually. ${ }^{1}$ Headaches are the most common, persistent, and debilitating sequelae of mTBI, with estimates of up to $90 \%$ prevalence following mTBI. ${ }^{2}$ The costs associated with posttraumatic headache (PTH) are high, with poorer performance on neurocognitive testing, increased risk for compounded disability, and a protracted recovery. ${ }^{2-4}$

PTH can be quite variable in clinical characteristics and symp-

Received August 31, 2016; accepted after revision November 16.

From the Department of Neuroradiology (L.M.A.), Barrow Neurological Institute, Phoenix, Arizona; Department of Radiology (J.D.), Division of Neuroradiology, University of Pittsburgh Medical Center, Pittsburgh, Pennsylvania; and East Valley Diagnostic Imaging (S.F.), Banner Health and Hospital System, Mesa, Arizona.

Please address correspondence to Lea Alhilali, MD, Barrow Neurological Institute, Department of Neuroradiology, 350 W Thomas Rd, Phoenix, AZ 85013; e-mail: lalhilali@sniweb.net

http://dx.doi.org/10.3174/ajnr.A5073 tomatology, with patients presenting with tension-type, migraine and clusterlike, cervicogenic, and mixed headaches. ${ }^{5}$ Despite the different symptoms associated with the various types of PTH, most studies evaluating PTH did not distinguish among the different PTH groups, focusing merely on the presence or absence of PTH. ${ }^{5-7}$ However, there is mounting evidence that important differences exist in the pathophysiology underlying posttraumatic migraines (PTMs) and the remaining types of PTH. ${ }^{2,8}$ Disrupted ionic homeostasis and altered electrophysiologic patterns are unique to PTM. ${ }^{8}$ Furthermore, PTM alone demonstrates a strong correlation with both decreased performance on neurocognitive testing and a delayed recovery. ${ }^{2,3}$

The mechanism underlying PTM following mTBI is poorly understood. At a molecular level, there are similarities between the pathophysiologic changes after mTBI and those seen with atraumatic migraines, including abnormalities of cellular sodium/ potassium homeostasis and increases in cellular markers of hypoxia. ${ }^{9}$ At a macroscopic level, abnormalities in cerebral blood flow 
and subcortical connectivity ${ }^{2,10}$ after mTBI mirror changes seen in patients with nontraumatic migraine. Structurally, similar regions of gray matter volume loss have been found in the frontal regions in patients with both nontraumatic migraines and chronic PTM. ${ }^{7,11}$ However, no studies have yet evaluated the role of white matter injury in the development of PTM, despite white matter injuries being seen with nontraumatic migraine, especially in the corpus callosum (CC). ${ }^{12,13}$

Given increasing awareness of white matter injuries underlying nontraumatic migraine, we sought to determine whether there was a relationship between white matter injury and PTM in patients with $\mathrm{mTBI}$. Previous studies have demonstrated that distinct white matter injuries after mTBI result in different and unique postconcussion symptoms, including sleep-wake disturbances, vestibulopathy, and ocular convergence insufficiency. ${ }^{14,15}$ We therefore hypothesized that a unique axonal injury pattern also underlies PTM, with a distinct injury pattern in patients with mTBI with posttraumatic migraine symptoms. Thus, the purpose of this study was to determine whether a central axonal injury underlies PTM after mTBI by using a Tract-Based Spatial Statistics (TBSS; http://fsl.fmrib.ox.ac.uk/fsl/fslwiki/TBSS) analysis of diffusion tensor imaging.

\section{MATERIALS AND METHODS \\ Subjects}

Our institutional review board approved this study, with a waiver of informed consent. All studies included were performed as the standard of care, and retrospectively reviewed.

We searched our electronic medical records to retrospectively identify MR imaging studies performed with DTI for mTBI. Radiology reports from March 1, 2006, to January 1, 2014, were searched with the keywords "concussion" and "diffusion-tensor imaging." Inclusion criteria for patients and controls were the following: 10-50 years of age, witnessed closed head trauma, no focal neurologic deficit, no loss of consciousness of $>1$ minute, posttraumatic amnesia of $<30$ minutes, and English language proficiency. Exclusion criteria for patients and controls were the following: a history of a neuropsychiatric illness (2 patients) or substance abuse (3 patients), abnormal CT or conventional MR imaging findings ( 3 patients), lack of DTI (4 patients) or neurocognitive assessment ( 6 patients), or the initial Total Symptom Score being zero (3 patients).

Patients underwent neurocognitive testing with the Immediate Post-Concussion Assessment Cognitive Test, which also produces a Total Symptom Score by using a 7-point Likert survey over 22 categories. Individuals were classified as patients if they had migraine headaches based on the International Headache Society guidelines ${ }^{16}$ following a postconcussion clinical examination (Table 1). The remaining patients with mTBI were selected as controls. Postconcussive headache was not an exclusion criterion for controls as long as it did not meet the International Headache Society guidelines for migraines. "Time to recovery" was defined as the patient stating that he or she was asymptomatic or the Total Symptom Score being zero (clinical examination performed by a clinical neuropsychologist with 14 years of experience in treating patients with concussion and chart review by 2 Certificate of
Table 1: International Headache Society guidelines for classification of migraines and definition of migraine aura ${ }^{18}$

Definition of migraine

At least 5 attacks fulfilling the following criteria:

1) Headache lasting 4-72 hr

2) Headache with at least 2 of the following characteristics: Unilateral location

Pulsating quality

Moderate or severe pain intensity

Aggravation by or causing avoidance of routine physical activity

3) During the headache, at least 1 of the following:

Nausea and/or vomiting

Photophobia or phonophobia

4) Not attributed to another disorder

Definition of aura

At least 1 of the following, but no motor weakness:

1) Fully reversible visual symptoms, including positive features (eg, flickering lights) and/or negative features (ie, loss of vision)

2) Fully reversible sensory symptoms, including positive features (ie, pins and needles) and/or negative features (ie, numbness)

3) Fully reversible dysphasic speech disturbance

Added Qualification-certified neuroradiologists [L.M.A., S.F.] with 5 years of experience).

\section{DTI and Conventional MR Imaging Assessments}

Conventional MR imaging and DTI were performed with a $1.5 \mathrm{~T}$ unit (Signa; GE Healthcare, Milwaukee, Wisconsin) and a standard head coil. Despite the relatively long span of this study, all patients and controls included in this study underwent the same imaging sequences on the same system as follows: sagittal and axial T1-weighted (TR, $600 \mathrm{~ms}$; TE, minimum; section thickness, $5 \mathrm{~mm}$; NEX, 1), axial proton-density-weighted (TR, 2000-2500 ms; TE, minimum; section thickness, $5 \mathrm{~mm}$; NEX, 1), T2weighted (TR/TE, 2000-2500/84-102 ms; section thickness, 5 $\mathrm{mm}$; NEX, 1), fluid-attenuated inversion recovery (TR/TE, 900010,000/149 ms; TI, $2200 \mathrm{~ms}$ ), and diffusion-weighted (single-shot echo-planar sequence; TR, 10,000 ms; TE, minimum; section thickness, $5 \mathrm{~mm}$; matrix, $128 \times 128)$. T2*-weighted gradient recalled-echo (TR/TE, 4400/21 ms; NEX, 1; flip angle, $90^{\circ}$; section thickness, $3 \mathrm{~mm}$ ) or susceptibility-weighted (TR/TE, 37/23 ms; NEX, 1; flip angle, $15^{\circ}$; section thickness, $2.4 \mathrm{~mm}$ ) sequences were performed. FOV ranged from 200 to $240 \mathrm{~mm}$.

DTI was performed with a single-shot echo-planar sequence (TR/TE, 4000/80 ms; NEX, 2; section thickness, 5 mm; matrix, $128 \times 128$; FOV, $260 \mathrm{~mm}$ ). Diffusion gradients were set in 25 noncollinear directions by using $2 \mathrm{~b}$-values $(b=0$ and 1000 $\left.\mathrm{s} / \mathrm{mm}^{2}\right)$.

\section{TBSS Analysis}

TBSS from FSL (http://www.fmrib.ox.ac.uk/fsl) was used to analyze DTI by using a skeleton-based approach to resolve alignment inaccuracies. ${ }^{17}$ TBSS works well for studies that include both adults and children, in which variability in head size or FOV may hinder other voxelwise techniques. Accordingly, TBSS has been used in studies of white matter development spanning pediatric and adult populations ${ }^{18}$ and to evaluate pathologies in both children and adults, such as mTBI. ${ }^{14,15}$ Analysis was performed as follows: frac- 
Table 2: Comparison of demographic and clinical characteristics of patients with $\mathbf{m T B I}$ with and without PTMs

\begin{tabular}{lccc}
\hline & + PTM & - PTM & P Value $^{\mathbf{a}}$ \\
\hline Mean age (range) (yr) & $17.6(10-38)$ & $19.7(12-47)$ & .26 \\
No. of males & $39(67 \%)$ & $12(71 \%)$ & 1.00 \\
Median time to presentation (range) (day) & $22(2-506)$ & $24(1-261)$ & .79 \\
Prior concussion (No.) & $24(41 \%)$ & $8(47 \%)$ & .56 \\
Sports injury (No.) & $34(59 \%)$ & $8(47 \%)$ & .42 \\
Mean ImPACT Total Symptom Score & $36.1(1-97)$ & $20.8(1-74)$ & .01 \\
$\quad$ (percentile) (range) & & & \\
Mean Verbal Memory Score (percentile) (range) & $30.2(1-99)$ & $37.5(7-92)$ & .20 \\
Mean Visual Memory Score (percentile) (range) & $28.1(1-97)$ & $36.5(1-88)$ & .13 \\
Mean reaction time (percentile) (range) & $34.6(1-95)$ & $43.1(1-94)$ & .18 \\
Mean processing speed (percentile) (range) & $33.5(1-98)$ & $47.7(1-94)$ & .05 \\
Median time to recovery (range) (week) & $51.9(1-252)$ & $39.4(3-194)$ & .50 \\
\hline
\end{tabular}

a $P$ values were 2 -tailed and calculated with the use of an unpaired $t$ test for continuous variables and a Fisher exact test for categoric variables.

${ }^{\mathrm{b}}$ ImPACT indicates Immediate Post-Concussion Assessment and Cognitive Test. Scores are percentiles determined by normative data from baseline testing of $>17,000$ athletes as part of their presport participation with percentile information accounting for both sex and age.

tional anisotropy (FA) or diffusivity images, including mean diffusivity, axial diffusivity, and radial diffusivity, were aligned into a common space by using the FMRIB Nonlinear Registration Tool (FNIRT; http://fsl.fmrib.ox.ac.uk/fsl/fslwiki/FNIRT), which uses a B-spline representation of the registration warp field. A mean FA image was then created and thinned to create a mean FA skeleton. The FA skeleton was thresholded for FA $\geq 0.2$ to suppress areas of extremely low FA or regions with considerable variability. Each subject's aligned FA data were then projected onto this skeleton, and the resulting data were fed into voxelwise cross-subject statistics.

A Monte Carlo permutation test (5000 permutations) was used with a general linear model and threshold-free cluster enhancement (significance at $P<.05$, family-wise error-corrected for multiple comparisons). We used a general linear model to determine areas of significantly different DTI metrics in patients with mTBI compared with controls, adjusting for covariates of age, sex, and prior concussion. Processing was performed by 2 Certificate of Added Qualification-certified neuroradiologists with 5 years of neuroradiology experience (L.M.A., S.F.) and a physician with 3 years of image analysis experience (J.D.).

\section{ROI Data Analysis}

ROI analysis was performed to quantify DTI values in regions of significant difference localized with TBSS. ROI analysis was based on the original TBSS mean skeleton overlaid with regions of significant difference in DTI metrics between patients and control subjects (corrected, voxelwise). The clusters with significant differences between patients and controls were used as ROIs for further analyses. FA or diffusivity values of patients and control subjects were then extracted in an automated fashion from each of these ROIs. Data extraction was limited to the voxels contributing to the TBSS skeleton to minimize partial volume effects. Values between patients and controls were compared with a 2-sample $t$ test. Correlation of FA or diffusivity values extracted from the ROI with continuous variables was performed with the Pearson correlation coefficient. The Cohen $\mathrm{d}$ was used to assess effect size. Analysis was performed by 2 Certificate of Added Qualification-certified neuroradiologists with 4 years of experience in image analysis (L.M.A., S.F.).

\section{Additional Statistical Analyses}

Statistical analysis of proportions and means in the demographic data was performed with the Fisher exact test and the unpaired 2-tailed $t$ test, respectively. $P<$ .05 was considered a statistically significant difference. Analysis was performed by a physician with postgraduate statistics training (L.M.A.).

\section{RESULTS}

Subjects

Seventy-four subjects with mTBI were included (51 males, 23 females; mean age, 18 years; range, $10-47$ years). The median time from injury to presentation was 20 days (range, 0-506 days). Sports injury was the most common mechanism of trauma (43 patients, 57\%), followed by motor vehicle collision ( 9 patients, 12\%). Of the 74 patients with mTBI included, 58 had PTMs. Controls consisted of 17 patients with mTBI without PTMs.

Patients with mTBI with migraines had a significantly higher Total Symptom Score than those without migraines $(P=.01)$. Patients with PTMs trended toward poorer performance on tests of processing speed $(P=.05)$. Comparison of the demographic and clinical characteristics between subjects with mTBI with and without PTMs is summarized in Table 2.

\section{DTI Assessment with TBSS}

Voxelwise analysis in patients with mTBI with and without PTM demonstrated that those with PTM had significantly lower FA in the CC and fornix/septohippocampal circuit (FSHC) than those without PTM (Fig 1). There was a trend toward increased mean diffusivity $(P=.10)$ in the $\mathrm{CC}$ of those with compared with those without PTM. There were no regions where patients with mTBI without PTM had lower FA or higher mean diffusivity than those with mTBI with PTM. No significant differences were seen between the 2 groups in axial or radial diffusivity.

\section{DTI Assessment with ROI Analysis}

FA in regions of abnormality in the FSHC identified by TBSS correlated with performance on neurocognitive tests of visual memory $(r=0.325, P=.01)$. Comparison of FA in ROIs for patients with $\mathrm{mTBI}$ with and without PTM are summarized in Tables 3 and 4 .

\section{DISCUSSION}

Using voxel-based analysis of DTI, we found injury to the CC and FSHC in patients with mTBI with PTM compared with mTBI without PTM. Injuries to the FSHC correlated with decreased performance on neurocognitive testing in the realm of visual memory.

Previous studies have used spectroscopy or volumetric analysis of gray matter to evaluate changes associated with PTH. These studies have found evidence of multifocal neuronal injury in the frontal and parietal regions, with spectroscopy and gray matter volume loss in the dorsolateral prefrontal cortex. ${ }^{7,19}$ However, 


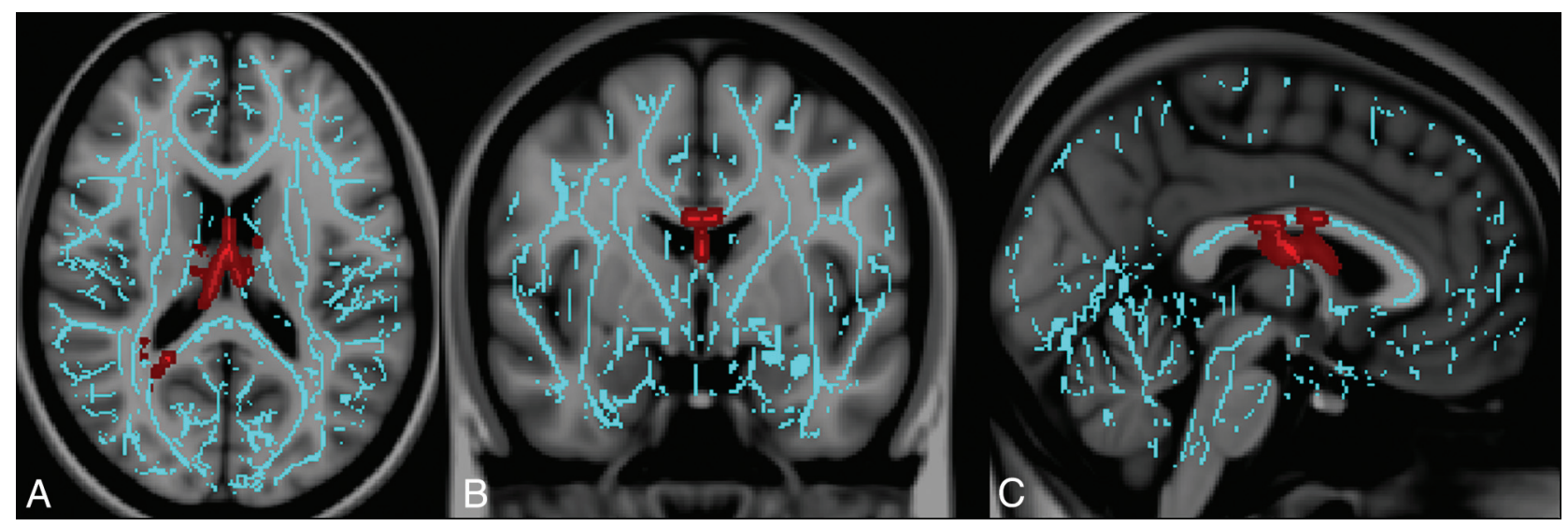

FIG 1. Regions of injury involving the corpus callosum and fornix/septohippocampal circuit among patients with posttraumatic migraine headaches. Images derived from Tract-Based Spatial Statistics results and rendered on T1-weighted images from the Montreal Neurological Institute atlas show common regions of injury involving the corpus callosum and fornix/septohippocampal circuit in patients with posttraumatic migraine headaches. ROIs involving the FSHC and CC corresponding to voxels of significant difference in FA $(P<.05$ corrected for multiple comparisons) between controls (subjects with mTBI without PTM) and patients with mTBI with PTM in are shown overlaid on the white matter skeleton (blue). ROls corresponding to voxels of significantly decreased FA in patients with mTBI with PTM are shown in red in the axial (A), coronal $(B)$, and sagittal $(C)$ planes.

Table 3: Comparison of FA in ROIs for patients with mTBI with and without PTMs

\begin{tabular}{|c|c|c|c|}
\hline & $\begin{array}{l}\text { Patients with } \\
\text { mTBI + PTMs }\end{array}$ & $\begin{array}{l}\text { Patients with } \\
\text { mTBI - PTMs }\end{array}$ & $\begin{array}{c}P \text { Value }^{a} \\
\left(d^{b}\right)\end{array}$ \\
\hline $\begin{array}{l}\text { Mean FA } \\
\text { value, CC } \\
(95 \% \mathrm{Cl})\end{array}$ & $0.688(0.581-0.793)$ & $0.721(0.615-0.823)$ & $.028(.680)$ \\
\hline $\begin{array}{l}\text { Mean FA } \\
\text { value, FSHC } \\
(95 \% \mathrm{Cl})\end{array}$ & $0.315(0.284-0.344)$ & $0.327(0.298-0.356)$ & $.045(.827)$ \\
\hline
\end{tabular}

${ }^{a} P$ values were 2 -tailed and calculated with an unpaired $t$ test.

${ }^{\mathrm{b}}$ The value of the Cohen $\mathrm{d}$.

Table 4: Correlation of FA with clinical findings for ROls in patients with $\mathrm{mTBI}$ and PTMs

\begin{tabular}{lcc}
\hline \multicolumn{1}{c}{ Variable } & $\begin{array}{c}\text { Correlation } \\
\text { with Mean } \\
\text { FA Value } \\
\text { CC ROI }\end{array}$ & $\begin{array}{c}\text { Correlation } \\
\text { with Mean } \\
\text { FA Value } \\
\text { FSHC ROI }\end{array}$ \\
\hline Age $^{\mathrm{a}}(P \text { value })^{\mathrm{b}}$ & $0.092(.49)$ & $-0.141(.29)$ \\
Verbal Memory Score $(P \text { value })^{\text {Visual Memory Score }(P \text { value })}$ & $-0.120(.37)$ & $-0.167(021)$ \\
Processing-speed score $(P$ value $)$ & $-0.122(.36)$ & $0.325(.01)$ \\
Reaction time score $(P$ value $)$ & $-0.133(.32)$ & $-0.143(.28)$ \\
Symptom Severity Score $(P$ value $)$ & $-0.072(.59)$ & $-0.113(.40)$ \\
Time to recovery $(P$ value $)$ & $0.156(.24)$ & $-0.015(.91)$ \\
\hline
\end{tabular}

${ }^{a}$ Correlation was performed with the Pearson correlation coefficient

${ }^{\mathrm{b}}$ Two-tailed $P$ value for the Pearson correlation coefficient.

these studies did not directly interrogate the microstructural integrity of the white matter, despite evidence that white matter injury is a common feature of both mTBI and migraines. ${ }^{11,14,15}$

Our DTI-based evaluation of white matter microstructure in PTM demonstrated common abnormalities in 2 major regions: the CC and FSHC. Both of these regions have been implicated in the pathogenesis of nontraumatic migraines. DTI abnormalities in the CC are commonly seen in nontraumatic migraine, in which they are associated with a more chronic disease course, greater headache frequency, and comorbid neuropsychiatric conditions. ${ }^{21}$ It is therefore not surprising that FA in the CC in our study trended toward an inverse correlation with time to recovery. The role of the FSHC in migraines is less clear, but it has been implicated in cortical spreading depression, a propagating, temporary loss of membrane potential in neurons thought to play a part in the pathophysiology of migraines. ${ }^{20,22}$

The FSHC contains serotonergic pathways. Multiple studies have documented the important role of central serotonergic systems in the neurobiology of migraine, with abnormalities seen in serotonin (5-HT) reuptake/metabolism and 5-HT-related signaling both during and between migraine episodes. In fact, inadequate signaling along important pain and limbic 5-HT pathways is thought to be a primary component of the pathobiology of migraine headache. ${ }^{23}$ The FSHC is part of the serotonin projections along the median raphé nucleus-dorsal hippocampal pathway. Fibers of this tract that run through the FSHC are very thin, with small varicosities, and are the most susceptible to injury. ${ }^{24}$

Treatment of migraine headaches in the nontraumatic setting is based mainly on the assumption that abnormalities of serotonin signaling result in an inflammatory process that can be inhibited with serotonin receptor agonists. The discovery of sumatriptan, a full agonist of the serotonin receptors $\left(5-\mathrm{HT}_{1 \mathrm{~b}, 1 \mathrm{~d}, 1 \mathrm{f}}\right)$ revolutionized the treatment of migraines in the general population. ${ }^{23}$ Pilot studies of sumatriptan use in PTM have demonstrated efficacy in the mTBI population in the acute setting. ${ }^{25,26}$ The FSHC and CC both express the $5-\mathrm{HT}_{1 \mathrm{a}}$ receptor, ${ }^{23,27-29}$ for which serotonin is a partial agonist.

Although quantitative use of DTI metrics in the clinical setting is currently limited by the lack of universally accepted normative data, demonstrating injured regions associated with PTM is the first step toward an individual diagnostic tool. Furthermore, knowledge that injuries to these pathways result in migraines may help radiologists recognize the relationship between macroscopic lesions in these areas (eg, macroscopic trauma, multiple sclerosis) and patient headache symptomatology.

Our study has limitations. Our evaluation was a retrospective, single-institution study with a moderate sample size. Additionally, the number of controls in our population was small, largely because our controls were not healthy controls, but rather patients with mTBI without migraine symptoms. As a result of the 
very high prevalence of posttraumatic migraines among patients with $\mathrm{mTBI},{ }^{30}$ it is very difficult to obtain a large cohort of patients with mTBI without posttraumatic migraines. Accordingly, the findings should be corroborated with a multicenter, prospective study. Furthermore, because most patients with mTBI do not undergo imaging, there is a possible selection bias toward patients with more severe injuries. However, these are also the more symptomatic patients, in whom imaging biomarkers are most needed.

\section{CONCLUSIONS}

White matter injuries in patients with mTBI with PTM may indicate that axonal injury can result in migraine symptomatology. White matter injuries in patients with PTM were in regions implicated in the pathophysiology of nontraumatic migraines, which may help elucidate the biologic underpinning of disease processes, direct treatment, and guide future research.

\section{REFERENCES}

1. Gardner RC, Yaffe K. Epidemiology of mild traumatic brain injury and neurodegenerative disease. Mol Cell Neurosci 2015;66(pt B): 75-80 CrossRef Medline

2. Kontos A, Elbin R, Lau B, et al. Posttraumatic migraine as a predictor of recovery and cognitive impairment after sports-related concussion. Am J Sports Med 2013;41:1497-504 CrossRef Medline

3. Mihalik JP, Register-Mihalik J, Kerr ZY, et al. Recovery of posttraumatic migraine characteristics in patients after mild traumatic brain injury. Am J Sports Med 2013;41:1490-96 CrossRef Medline

4. Carlson $\mathrm{K}$, Taylor B, Hagel E, et al. Headache diagnoses among Iraq and Afghanistan war veterans enrolled in VA: a gender comparison. Headache 2013;53:1573-82 CrossRef Medline

5. D'Onofrio F, Russo A, Conte F, et al. Post-traumatic headaches: an epidemiological overview. Neurol Sci 2014;35(suppl 1):203-06 CrossRef Medline

6. Packard RC. Chronic post-traumatic headache: associations with mild traumatic brain injury, concussion, and post-concussive disorder. Curr Pain Headache Rep 2008;12:67-73 CrossRef Medline

7. Obermann M, Nebel K, Schumann C, et al. Gray matter changes related to chronic posttraumatic headache. Neurology 2009;73: 978-83 CrossRef Medline

8. Kontos AP, Reches A, Elbin RJ, et al. Preliminary evidence of reduced brain network activation in patients with post-traumatic migraine following concussion. Brain Imaging Behav 2016;10:594-603 CrossRef Medline

9. Harris JL, Yeh HW, Choi IY, et al. Altered neurochemical profile after traumatic brain injury: (1)H-MRS biomarkers of pathological mechanisms. J Cereb Blood Flow Metab 2012;32:2122-34 CrossRef Medline

10. Tang L, Ge Y, Sodickson DK, et al. Thalamic resting-state functional networks: disruption in patients with mild traumatic brain injury. Radiology 2011;260:831-40 CrossRef Medline

11. Bashir A, Lipton RB, Ashina S, et al. Migraine and structural changes in the brain: a systemic review and meta-analysis. Neurology 2013; 81:1260-68 CrossRef Medline

12. Yu D, Yuan K, Qin W, et al. Axonal loss of white matter in migraine without aura: a tract-based spatial statistics study. Cephalgia 2013; 33:34-42 CrossRef Medline

13. Rocca MA, Pagani E, Colombo B, et al. Selective diffusion changes of the visual pathway in patients with migraine: a 3-T tractography study. Cephalgia 2008;28:1061-68 CrossRef Medline

14. Fakhran S, Yaeger K, Alhilali L. Symptomatic white matter changes in mild traumatic brain injury resemble pathologic features of early Alzheimer dementia. Radiology 2013;269:249-57 CrossRef Medline

15. Alhilali LM, Yaeger K, Collins M, et al. Detection of central white matter injury underlying vestibulopathy after mild traumatic brain injury. Radiology 2014;272:224-32 CrossRef Medline

16. Headache Classification Subcommittee of the International Headache Society. The International Classification of Headache Disorders: 2nd edition. Cephalalgia 2004;24(suppl 1):9-160 CrossRef Medline

17. Smith SM, Jenkinson M, Johansen-Berg H, et al. Tract-based spatial statistics: voxelwise analysis of multi-subject diffusion data. Neuroimage 2006;31:1487-505 CrossRef Medline

18. Giorgio A, Watkins KE, Chadwick M, et al. Longitudinal changes in grey and white matter during adolescence. Neuroimage 2010;49:94103 CrossRef Medline

19. Sarmento E, Moreira P, Brito C, et al. Proton spectroscopy in patients with post-traumatic headache attributed to mild head injury. Headache 2009;49:1345-52 CrossRef Medline

20. Bartsch T, Goadsby PJ. The trigeminocervical complex and migraine: current concepts and synthesis. Curr Pain Headache Rep 2003;7:371-76 CrossRef Medline

21. Li XL, Fang YN, Gao QC, et al. A diffusion tensor magnetic resonance imaging study of corpus callosum from adult patients with migraine complicated with depressive/anxious disorder. Headache 2011;51:237-45 CrossRef Medline

22. Moskowitz MA, Nozaki K, Kraig RP. Neocortical spreading depression provokes the expression of $\mathrm{c}$-fos protein-like immunoreactivity within trigeminal nucleus caudalis via trigeminovascular mechanisms. J Neurosci 1993;13:1167-77 Medline

23. Hamel E. Serotonin and migraine: biology and clinical implications. Cephalalgia 2007;27:1293-300 CrossRef Medline

24. Murphy DL, Andrews AM, Wichems $\mathrm{CH}$, et al. Brain serotonin neurotransmission: an overview and update with an emphasis on serotonin subsystem heterogeneity, multiple receptors, interactions with other neurotransmitter systems, and consequent implications for understanding the actions of serotonergic drugs. J Clin Psychiatry 1998;59(suppl 15):4-12 Medline

25. McCrory P, Heywood J, Ugoni A. Open label study of intranasal sumatriptan (Imigran) for footballer's headache. Br J Sports Med 2005;39:552-54 CrossRef Medline

26. Abend NS, Nance ML, Bonnemann C. Subcutaneous sumatriptan in an adolescent with acute posttraumatic headache. J Child Neurol 2008;23:438-40 CrossRef Medline

27. Hawthorne AL, Hu H, Kundu B, et al. The unusual response of serotonergic neurons after CNS injury: lack of axonal dieback and enhanced sprouting within the inhibitory environment of the glial scar. J Neurosci 2011;31:5605-16 CrossRef Medline

28. Pacheco J, Beevers CG, Benavides C, et al. Frontal-limbic white matter pathway associations with the serotonin transporter gene promoter region (5-HTTLPR) polymorphism. J Neurosci 2009;29: 6229-33 CrossRef Medline

29. Caspers S, Schleicher A, Bacha-Trams M, et al. Organization of the human inferior parietal lobule based on receptor architectonics. Cereb Cortex 2013;23:615-28 CrossRef Medline

30. Lucas S, Hoffman JM, Bell KR, et al. A prospective study of prevalence and characterization of headache following mild traumatic brain injury. Cephalalgia 2014;34:93-102 CrossRef Medline 